\title{
Contralateral Delayed Acute Subdural Hematoma Following Evacuation of a Chronic Subdural Hematoma with Burr-Hole Craniotomy and Continuous Closed System Drainage: Case Report and Literature Review
}

\author{
Rong-Dar Tzou ${ }^{1,2}$, Feng-Ji Tsai ${ }^{* 1,2,3}$, Tai-Hsin Tsai ${ }^{1,2,3}$, Yu-Feng Su ${ }^{1,2}$ and Sui-Sum Kung ${ }^{1}$ \\ ${ }^{1}$ Division of Neurosurgery, Department of Surgery, Kaohsiung Medical University Hospital, Kaohsiung Medical Uni- \\ versity, Kaohsiung, Taiwan \\ ${ }^{2}$ Faculty of Medicine, College of Medicine, Kaohsiung Medical University, Kaohsiung, Taiwan \\ ${ }^{3}$ Institute of Medicine, Kaohsiung Medical University, Kaohsiung, Taiwan
}

${ }^{*}$ Corresponding author: Feng-Ji Tsai, M.D, Division of Neurosurgery, Department of Surgery, Kaohsiung Medical University Hospital, 100 Tzyou $1^{\text {st }}$ Road, Kaohsiung, Taiwan 80708, Fax: 886-7-3215039, Tel: 886-73215049, E-mail: 1srtsai0312@gmail.com

Citation: Rong-Dar Tzou, Feng-Ji Tsai, Tai-Hsin Tsai, Yu-Feng Su, Sui-Sum Kung (2016) Contralateral Delayed Acute Subdural Hematoma Following Evacuation of a Chronic Subdural Hematoma with Burr-Hole Craniotomy and Continuous Closed System Drainage: Case Report and Literature Review. J Case Rep Stud 4(2): 207. doi: 10.15744/2348-9820.4.207

Received Date: January 12, 2016 Accepted Date: April 16, 2016 Published Date: April 19, 2016

\begin{abstract}
Chronic subdural hematoma (CSDH) is a relatively frequent problem in neurologic or neurosurgical practice. Treatments for CSDH are well established and provide positive postoperative outcome; however, some atypical postoperative complications can occur. This study reports a rare complication of delayed contralateral acute hematoma following the evacuation of a chronic subdural hematoma with burr-hole craniotomy and continuous closed system drainage. According to our research, our study is the first to report a delayed contralateral acute subdural hematoma following CSDH surgery.
\end{abstract}

Keywords: Delayed contralateral acute subdural hematoma; Chronic subdural hematoma; Burr-hole craniotomy; Closed system drainage; Complication

List of Abbreviations: CSDH: Chronic subdural hematoma; CASDH: Contralateral acute subdural hematoma; CT: Computed tomography

\section{Introduction}

Chronic subdural hematoma $(\mathrm{CSDH})$ is a relatively frequent problem in neurologic or neurosurgical practice [1]. The potential for recovery from the evacuation of a CSDH is relatively high. However, some postoperative complications may affect the postoperative course [2]. In this study, we report a rare complication of contralateral acute hematoma following the evacuation of a CSDH with burr-hole craniotomy and continuous closed system drainage.

\section{Case Presentation}

A 78-year-old man presented with weakness in his right-side extremities and gait disturbance. He had a medical history of chronic kidney disease, dementia, and hypertension. Upon admission, a neurological examination revealed right hemiparesis (muscle power, 4 of 5), poor orientation in person and place, and isocoria with normal light reaction. The results from hematological investigations, biochemistry tests, and coagulation profile were within normal ranges. Computed tomography (CT) of the head showed a hypodense subdural hematoma over the left frontoparietotemporal region, causing a midline shift to the right and compression of the ipsilateral lateral ventricle (Figure 1A). A left chronic subdural hematoma was diagnosed. Burr-hole evacuation of the hematoma with continuous closed system drainage was performed immediately.

On Day 5, CT of the head was performed and near complete evacuation of the hematoma was observed (Figure 1B). However, on Day 6, the patient had an altered level of consciousness following the sudden efflux of a large amount of subdural effusion from the drainage system. CT of the head revealed an acute subdural hematoma on the contralateral side, with a midline shift to the left and compression of the ipsilateral lateral ventricle (Figure 1C). Under general anesthesia, a craniotomy was made over the right 
frontotemporoparietal region, and the subdural hematoma was observed in the space between the dura and an inner membrane (Figure 2A). Following the removal of the hematoma, bleeding from the torn bridge vein was observed, and the bleeding vein was coagulated (Figure 2B). An intracranial pressure monitoring system provided measurements of intracranial pressure at a subdural level, and consistently low intracranial pressure $(-3-5 \mathrm{mmHg})$ was recorded. The second postoperative course was uneventful. The patient was discharged 20 days later with mild neurological deficit.

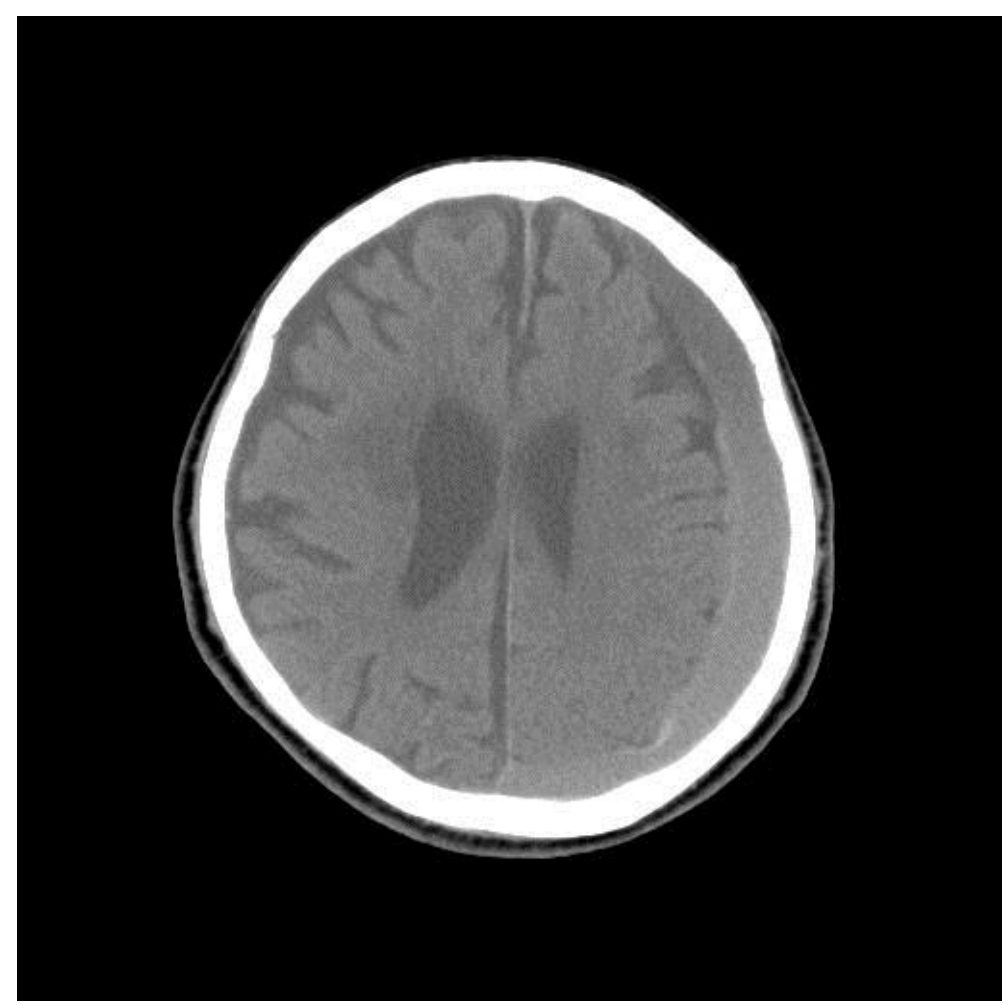

Figure 1A: Preoperative CT revealing a hypodense subdural hematoma over the left frontoparietotemporal region, causing a midline shift to the right and compression of the ipsilateral lateral ventricle

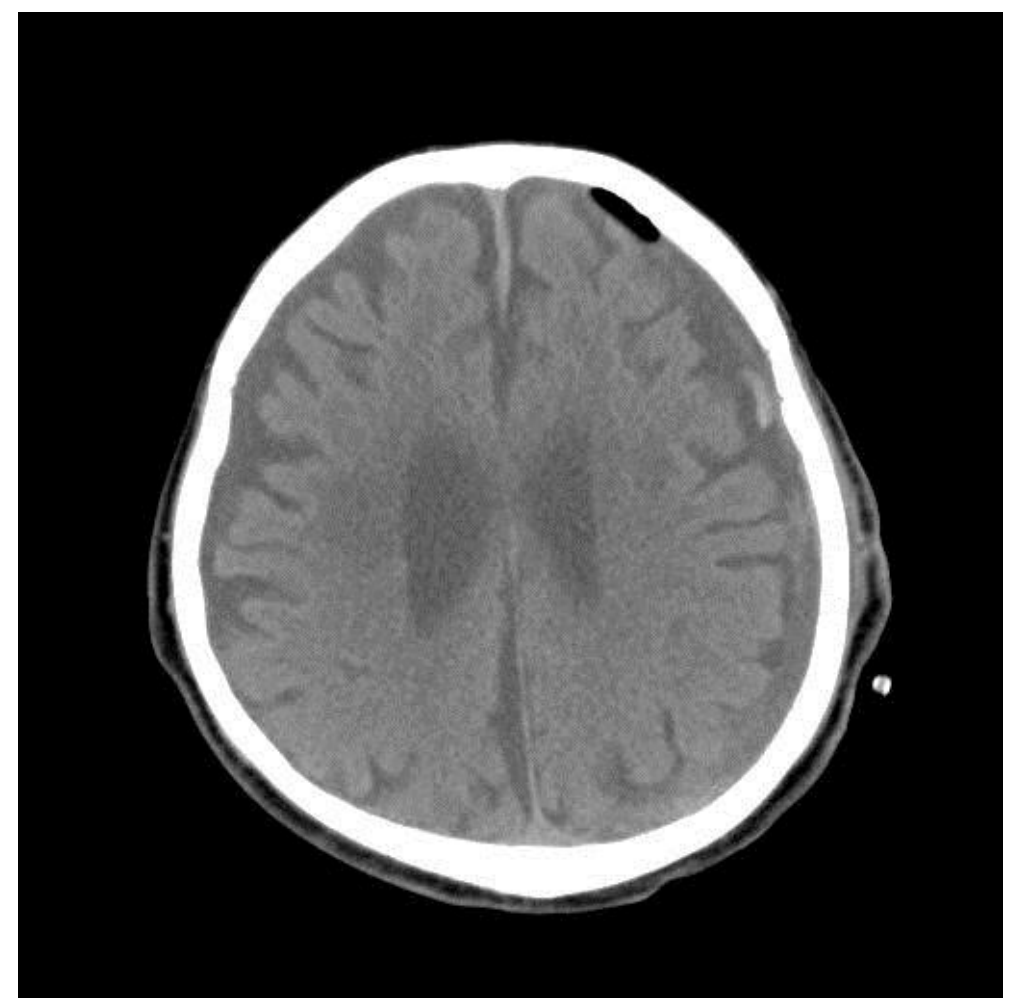

Figure 1B: First postoperative CT showing near complete evacuation of a left CSDH 


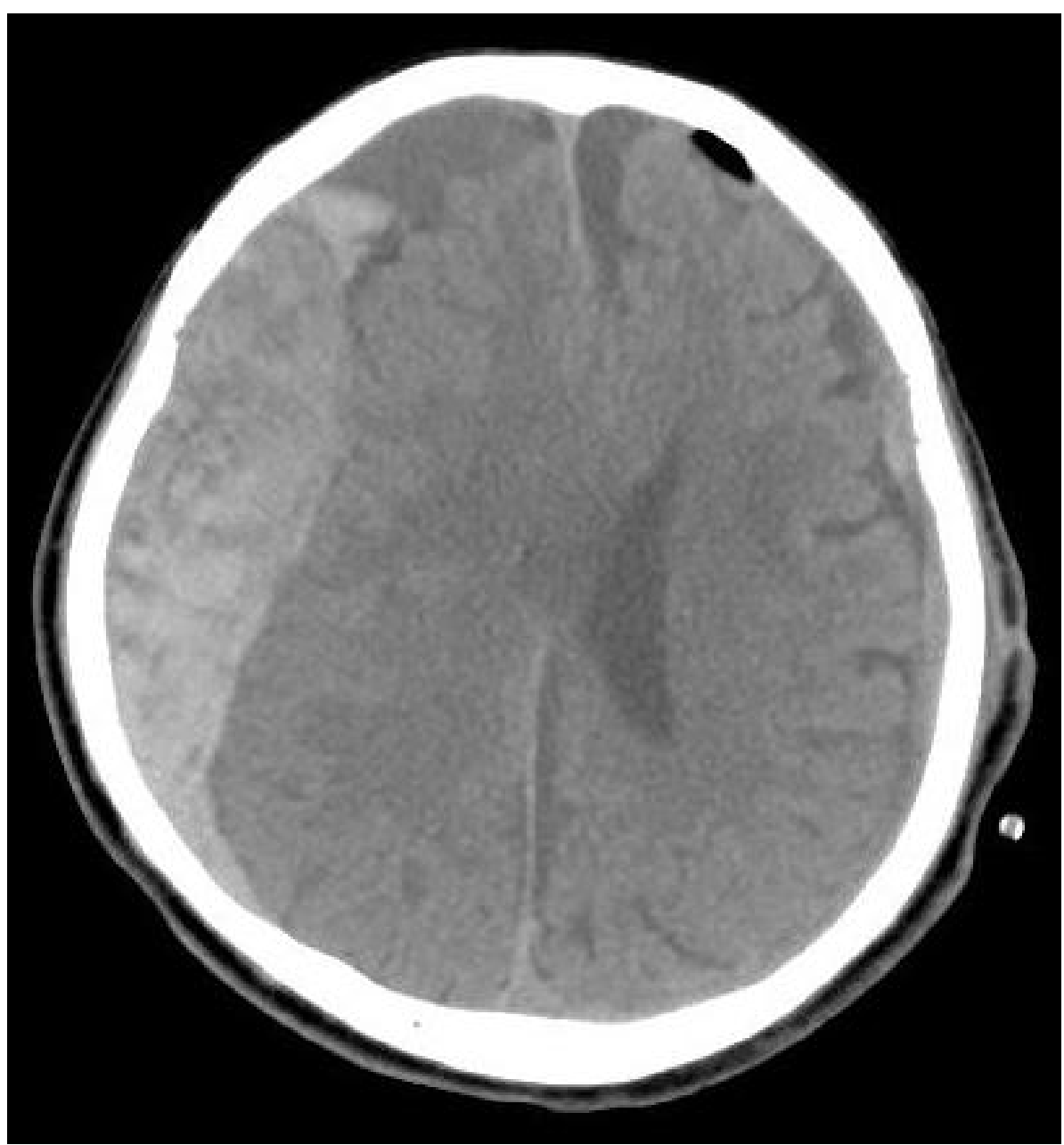

Figure 1C: Postoperative CT revealing a contralateral acute hematoma, suggesting a subdural hematoma with a midline shift to the left and compression of the ipsilateral lateral ventricle

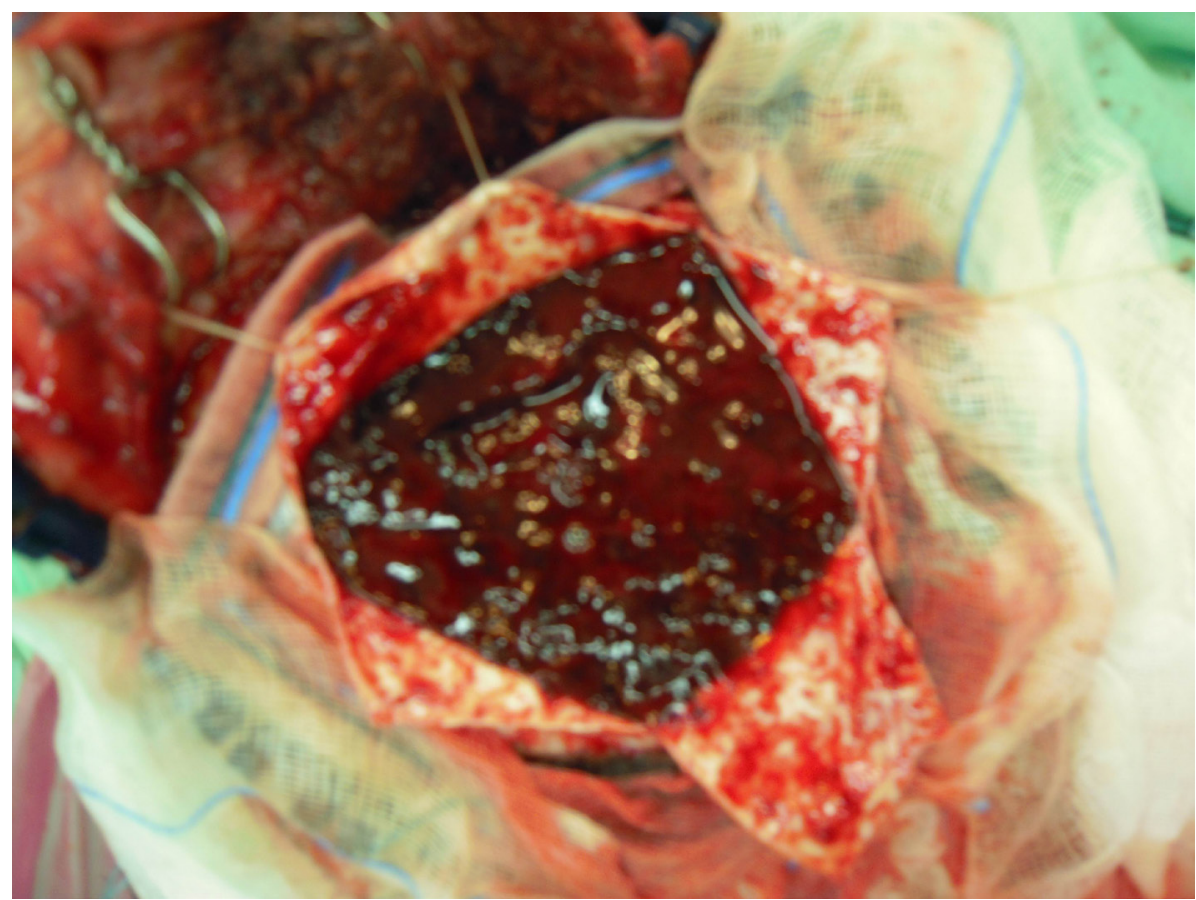

Figure 2A: Intraoperative photograph, showing the hematoma beneath the dura matter 


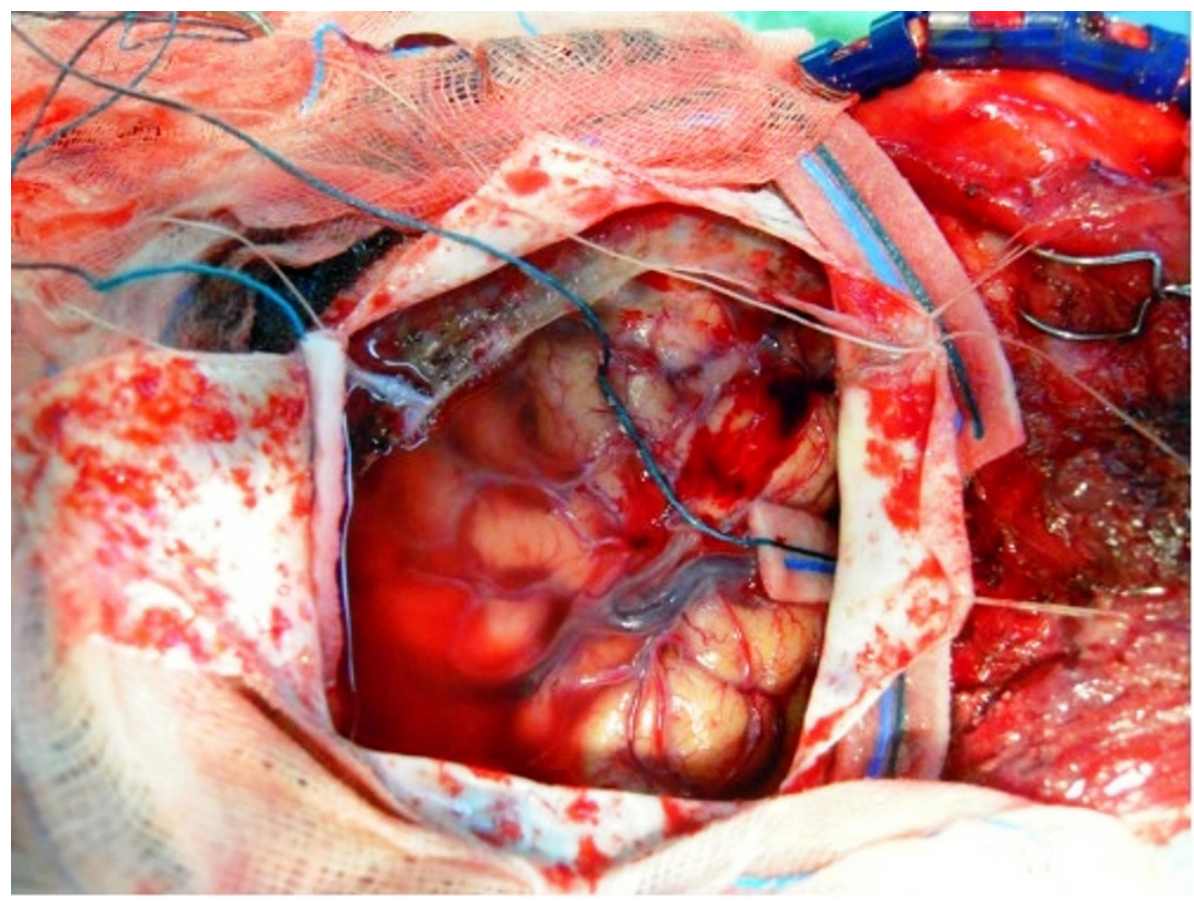

Figure 2B: The partial removal of an inner membrane below the hematoma

\section{Discussion}

A contralateral hematoma is a rare complication following the evacuation of a CSDH and has been well described previously (Table 1). The contralateral hematoma commonly occurs immediately. Rarely, the contralateral hematoma develops 6 days postsurgery. In this study, we report an unusual case of delayed contralateral acute subdural hematoma (CASDH) following the evacuation of a CSDH.

\begin{tabular}{|c|c|c|c|}
\hline Author/ year & Age/Gender & Type of hematoma & Mechanism \\
\hline $\begin{array}{c}\text { Eom KS } \text { et al. } \\
{[2](2009)}\end{array}$ & $78 / \mathrm{M}$ & AIDH & The formation of a contralateral hematoma \\
remains unclear.
\end{tabular}

AIDH: Acute Interdural Hematoma; AEDH: Acute Epidural Hematoma; ASDH: Acute Subdural Hematoma

Table 1: Contralateral acute hematoma following burr-hole craniotomy with continuous drainage

The pathophysiological mechanisms underlying a contralateral acute intracranial hematoma following burr-hole craniotomy with continuous closed system drainage are unclear. Several possible mechanisms could be responsible for contralateral acute hematoma development. The abrupt evacuation of a CSDH might result in a brain parenchyma shift, leading to contralateral bridge vein or cortical vein disruption [3-5]. Alternatively, a decrease in the intracranial pressure after the evacuation of a hematoma might disturb vascular autoregulation in patients suffering from chronic elevated intracranial pressure [3-7]. 
Hemorrhage into previously undetected areas of a contusion could also be responsible [3,4]. The rapid evacuation of a hematoma, disturbance of autoregulation, and presence of an undetected lesion are other possible causes. However, in our reported case, these mechanisms would not explain the presence of a delayed contralateral acute hematoma. Chronic intracranial hypotension is a risk factor for delayed acute hematoma. In conditions of intracranial hypotension, the brain cannot be fully supported, and the tensed bridge vein can easily damage.

Whether a contralateral acute hematoma can be avoided remains unclear. Minimal evidence exists to support the use of perioperative or postoperative methods in patients with chonic subdural hematoma undergoing burr-hole craniotomy and continuous system drainage. Modesti et al. [4] suggested using decompression with a slow rate of evacuation during the perioperative period to avoid an abrupt brain parenchyma shift. Kiymaz et al. [8] indicated that continuous drainage therapy for CSDH provides superior results to one-time drainage methods. However, intermittant drainage might provide an alterative procedure to continuous drainage to avoid the rapid evacuation of a hematoma postsurgery. An appropriate fluid supplement should be administered to avoid chronic intracranial hypotension.

According to our research, our study is the first to report a delayed CASDH following burr-hole craniotomy with continuous closed system drainage. Our results indicate that the slow decompression of a CSDH and careful regulation of intracranial hypotension could prevent a delayed contralateral acute hematoma.

\section{References}

1. Turgut M, Akalan N, Sağlam S (1998) A fatal acute subdural hematoma occurring after evacuation of "contralateral" chronic subdural hematoma. J Neurosurg Sci 42: 61-3

2. Eom KS, Kim TY, Park JT (2009) Contralateral acute interdural haematoma occurring after burr hole drainage of chronic subdural haematoma. Br J Neurosurg 23: $213-5$.

3. Su TM, Shih TY, Yen HL, Tsai YD (2001) Contralateral acute subdural hematoma occurring after evacuation of subdural hygroma: case report. J Trauma 50: $557-9$.

4. Modesti LM, Hodge CJ, Barnwell ML (1982) Intracerebral hematoma after evacuation of chronic extracerebral fluid collections. Neurosurgery 10: 689-93.

5. Park KJ, Kang SH, Lee HK, Chung YG (2009) Brain stem hemorrhage following burr hole drainage for chronic subdural hematoma-case report. Neurol Med Chir 49: 594-7.

6. Sousa J, Golash A, Vaz J, Chaudhary H (2004) Spontaneous intracerebral haemorrhage following evacuation of chronic subdural hematomas. J Clin Neurosci 11: 794-6.

7. Miyajima K, Hayashi N, Kurimoto M, Kuwayama N, Hirashima Y, et al. (2004) Idiopathic interdural hematoma looking like a "Chinese dumpling"--case report. Neurol Med Chir 44: 75-6.

8. Kiymaz N, Yilmaz N, Mumcu C (2007) Controversies in chronic subdural hematoma: continuous drainage versus one-time drainage. Med Sci Monit 13: CR2403.

9. Panourias IG, Skandalakis PN (2006) Contralateral acute epidural haematoma following evacuation of a chronic subdural haematoma with burr-hole craniostomy and continuous closed system drainage: a rare complication. Clin Neurol Neurosurg 108: 396-9.

10. Liang CL, Rau C-S, Lu K, Chen H-J (2001) Contralateral acute subdural haematoma after burr-hole for chronic subdural haematoma. Injury 32: $499-500$.

11. Harada K, Ohtsuru K, Nakayama K, Takagi S, Shigemori M, et al. (1992) Contralateral development of acute subdural hematoma following surgery for chronic subdural hematoma--case report. Neurol Med Chir 32: 969-71.

12. Nagata K, Ide T, Basugi N, Sezai Y (1986) Acute Subdural Hematoma after Irrigation of the Contralateral Chronic Subdural Hematoma in a Hemodialyzed Patient Case Report. Neurol Med Chir 26: 510-3.

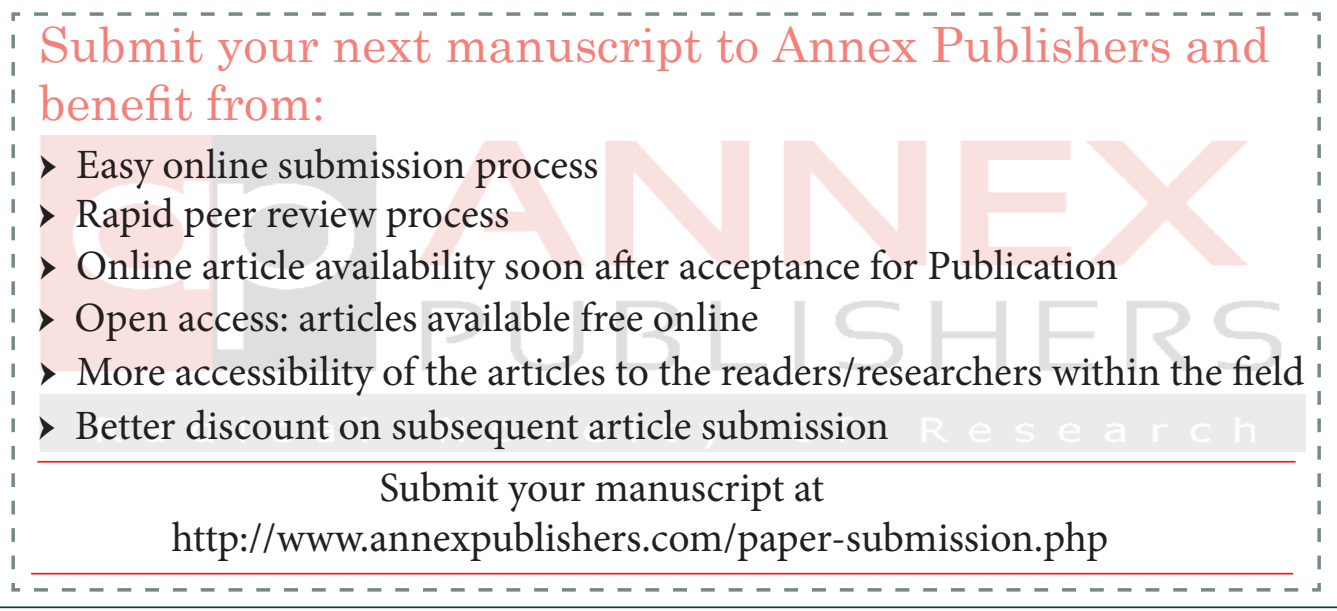

